\title{
Évaluation d'une atteinte cornéenne dans le cadre d'une maladie de Fabry par microscopie confocale
}

\author{
N. Mavrakanas, N. Tajouri-Morizur, A.A. Dosso \\ Clinique d'ophtalmologie, Hôpitaux Universitaires, Genève, Suisse. \\ Communication affichée au $113^{\mathrm{e}}$ Congrès de la Société Française d'Ophtalmologie, en mai 2007. \\ Correspondance : N. Mavrakanas, Clinique d'ophtalmologie, Hôpitaux Universitaires de Genève, 22 Rue Alcide Jentzer, 1211, Genève 14, Suisse. \\ E-mail : nikolaos.mavrakanas@hcuge.ch \\ Reçu le 1er octobre 2007. Accepté le 11 février 2008.
}

\section{Corneal findings in Fabry disease-related keratopathy using in vivo confocal microscopy}

N. Mavrakanas, N. Tajouri-Morizur, A.A. Dosso

\section{J. Fr. Ophtalmol., 2008; 31, 4: 416-418}

Aim: Description of corneal findings in Fabry disease-related keratopathy using in vivo confocal microscopy.

Observation: A 65-year-old man presenting with a cerebral ischemic lesion was referred to our clinic for ophthalmologic evaluation with a suspicion of inaugural Fabry disease. Slit lamp biomicroscopy showed whorl-like lines in the inferior cornea of both eyes. In vivo confocal microscopy revealed the presence of numerous hyperreflective intracellular inclusions in the corneal epithelium and stroma.

Discussion: The whorl-like lines are related to deposition and accumulation of glycosphingolipids in the epithelial and stromal layers of the cornea. This case is also interesting for the late manifestation of Fabry disease, showing the variability in the expression of this condition. Conclusion: In vivo confocal microscopy may be an important tool in the follow-up of Fabry disease-related keratopathy, enabling us to monitor the effect of enzyme replacement therapy.

Key-words: In vivo confocal microscopy, Fabry disease, cornea verticillata.

\section{Évaluation d'une atteinte cornéenne dans le cadre d'une maladie de Fabry par microscopie confocale}

But : Description des modifications cornéennes chez un patient atteint d'une maladie de Fabry par microscopie confocale.

Observation: Un homme, âgé de 65 ans, nous fut adressé dans le contexte d'une lésion ischémique cérébrale avec une suspicion de maladie de Fabry inaugurale, à la recherche de manifestations ophtalmologiques associées. La biomicroscopie du segment antérieur montrait dans les deux yeux une cornée verticillata. La microscopie confocale mit en évidence la présence de nombreuses inclusions hyper-réflectives au niveau épithélial et stromal.

Discussion : Les inclusions hyper-réflectives observées en microscopie confocale sont corrélées aux dépôts de glycosphingolipides dans les couches épithéliales et stromales de la cornée. Ce cas nous paraît intéressant également par le diagnostic tardif de la pathologie, ce qui démontre la variabilité de l'expression pathologique, même dans la maladie de Fabry.

Conclusion : La microscopie confocale pourrait avoir son intérêt dans le diagnostic et le suivi de cette déficience enzymatique et dans le contrôle de l'efficacité thérapeutique.

Mots-clés : Microscopie confocale, maladie de Fabry, cornée verticillata.

\section{INTRODUCTION}

La maladie de Fabry est une atteinte génétique ( $X$-linked), due à une activité déficiente d'un enzyme lysosomique, de la galactosidase $A$, induisant une erreur du métabolisme des glycosphingolipides. II s'agit d'une dysfonction multi-systémique, caractérisée par des angiokeratomes (lésions cutanées télangiéctasiques), d'une hypohydrose, d'une acroparésthesie, et des lésions vasculaires rénales, cardiaques et cérébrales [1]. Des atteintes oculaires sont fréquemment rencontrées, dont les plus courantes sont la cornée verticillata et le " haze » stromal [2-4].

Des microanévrismes conjonctivaux, une cataracte précoce, des dépôts au niveau de la capsule postérieure du cristallin, ainsi qu'une tortuosité du réseau vasculaire rétinien, ont également été rapportés [24]. Les signes cliniques oculaires ne sont pas constants, et la sévérité des manifestations cornéennes est plus importante chez les homozygotes que chez les hétérozygotes [2, 3, 5].

Nous présentons les observations microscopiques cornéennes chez un patient atteint d'une maladie de Fabry au moyen de la microscopie confocale.

\section{OBSERVATION}

Un homme, âgé de 65 ans, nous fut adressé dans un contexte de lésion 
ischémique cérébrale avec une suspicion de maladie de Fabry inaugurale, à la recherche de manifestations ophtalmologiques associées. Le diagnostic avait été posé ultérieurement par analyse génétique et un screening familial positif. L'anamnèse médicamenteuse était né- gative pour les médicaments susceptibles d'engendrer une cornée verticillata.

La meilleure acuité visuelle corrigée était de 20/25e des deux côtés. La biomicroscopie du segment antérieur mit en évidence la présence de lignes brunes sous-
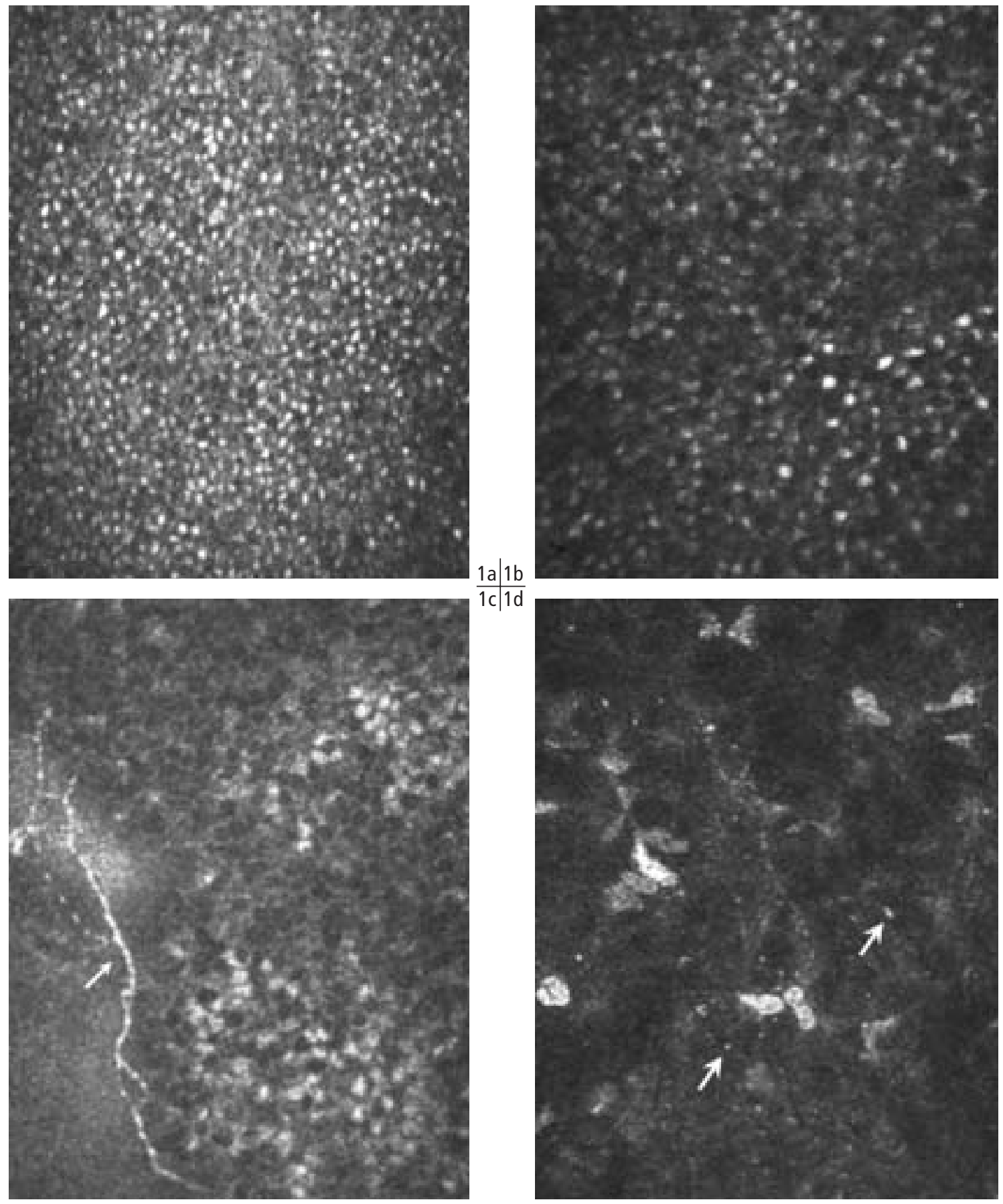

Figure 1 : Microscopie confocale. (a) et (b) Couche épithéliale. La densité d'accumulation des inclusions intracellulaires hyper-réflectives au niveau de l'épithélium basal est répartie de manière inhomogène. (a) La partie inférieure de la cornée est la plus touchée, alors que, (b) dans la partie supérieure, la densité d'accumulation est moindre. (c) Altérations des cellules épithéliales basales, un nerf sous-épithélial est visible (flèche) à côté d'une zone d'épithélium pathologique. (d) Présence d'inclusions hyper-réflectives dans le stroma (flèches). 
épithéliales dans le tiers inférieur de la cornée de manière bilatérale. En dehors d'une opalescence cristallinienne débutante et d'une tortuosité vasculaire rétinienne, l'examen ophtalmologique était frustre.

La microscopie confocale cornéenne a été réalisée avec le HRT II Rostock Cornea Module (Heidelberg Engineering $\mathrm{GmbH}$, Heidelberg, Allemagne). Des images séquentielles dérivées de balayage automatique et manuel de la cornée centrale et périphérique ont été obtenues. L'épaisseur de chaque section confocale était d'environ $10 \mu \mathrm{m}$, et la taille des images obtenues de $300 \times 300 \mu \mathrm{m}$. Elle montrait la présence de nombreuses inclusions hyper-réflectives au niveau épithélial et stromal. Des altérations au niveau du complexe cellules épithéliales basales/membrane de Bowman étaient aussi retrouvées (fig. 1). Par ailleurs, la morphologie, la réflectivité et la densité cellulaire de l'endothélium cornéen se sont révélées physiologiques, de même que l'aspect des nerfs cornéens.

\section{DISCUSSION}

Les inclusions hyper-réflectives observées en microscopie confocale sont corrélées aux dépôts de glycosphingolipides dans les couches épithéliales et stromales cornéennes [4, 6], en l'occurrence sans répercussion sur l'acuité visuelle chez ce patient avec néanmoins, un possible lien dans l'éblouissement décrit. Selon Mastropasqua et al. [7], les sphingolipides sont déposés dans la couche épithéliale et sous-épithéliale cornéenne, et la variation de la densité de ces accumulations donne I'aspect typique de la cornée verticillata. D'autre part, I'atteinte du complexe cellules épithéliales basales/ membrane de Bowman pourrait être la cause de la production d'un épithélium sus-jacent anormal ainsi que de la formation du haze stromal.

Ce cas nous paraît intéressant également par le diagnostic tout à fait tardif de la pathologie, qui démontre la variabilité d'expression pathologique possible, même dans la maladie de Fabry.

\section{CONCLUSION}

La microscopie confocale pourrait avoir son intérêt dans le diagnostic et le suivi de cette déficience enzymatique et dans le contrôle de l'efficacité thérapeutique. D'autres études qui comprendraient un nombre plus important de patients seraient nécessaires pour bien établir le rôle possible de cette méthode dans le cadre de la maladie de Fabry.

\section{RÉFÉRENCES}

1. Mehta A, Ricci R, Widmer U, Dehout F, Garcia de Lorenzo A, Kampmann $C$, et al. Fabry disease defined: baseline clinical manifestations of 366 patients in the Fabry Outcome Survey. Eur J Clin Invest 2004;34:236-42.

2. Orssaud C, Dufier J, Germain D. Ocular manifestations in Fabry disease: a survey of 32 hemizygous male patients. Ophthalmic Genet 2003;24:129-39.

3. Kono JO, Podskarbi T, Shin Y, Lanzl I. Oligosymptomatic cornea verticillata in a heterozygote for Fabry disease: a novel mutation in the alpha galactosidase gene. Cornea 2003;22:175-7.

4. Nguyen TT, Gin T, Nicholls K, Low M, Galanos J, Crawford A. Ophthalmological manifestations of Fabry disease: a survey of patients at the Royal Melbourne Fabry Disease Treatment Centre. Clin Experiment Ophthalmol 2005;33:164-8.

5. Fumex-Boizard L, Cochat P, Fouilhoux A, Guffon N, Denis P. Relation entre les manifestations ophtalmologiques et les atteintes générales chez dix patients atteints de la maladie de Fabry. J Fr Ophtalmol 2005;28:45-50.

6. Hirano K, Murata K, Miyagawa A, Terasaki H, Saigusa J, Nagasaka T, et al. Histopathologic findings of cornea verticillata in a woman heterozygous for Fabry's disease. Cornea 2001;20: 233-6.

7. Mastropasqua L, Nubile M, Lanzini M, Carpineto P, Toto L, Ciancaglini M. Corneal and conjunctival manifestations in Fabry disease: in vivo confocal microscopy study. Am J Ophthalmol 2006;141:709-18. 